ORIGINAL ARTICLE

\title{
3D Evaluation of Root Canal Configuration of Mandibular Premolars Using Cone Beam Computed Tomography (CBCT)
}

\author{
Muhammad Bilal Bashir', Imtiaz Ahmed ${ }^{2}$ \\ 'Department of Oral Biology, Dr. Ishrat-Ul-Ebad Khan Institute of Oral Health Sciences (DIKIOHS), Dow University of Health \\ Sciences, ${ }^{2}$ Department of Orthodontics, Dow University of Health Sciences, Karachi, Pakistan.
}

\begin{abstract}
Background: Cone Beam Computed Tomography (CBCT) provides 3D image, which can help in identifying more than one canals, accessory and lateral canals. This study aimed to evaluate the commonly found root canal morphology of mandibular premolars according to Vertucci's classification in the target Pakistani population using CBCT.
\end{abstract}

Methods: This is a cross-sectional study performed on $n=432$ mandibular premolars bilaterally. Patients who fulfilled the inclusion criteria were sent to radiology department for CBCT. The CBCT images were examined with the help of 3D planner version 2.0_2018 villa SM 2018 software. For the number of the roots, position of the root, canal bifurcation and Vertucci's classification of root canals Descriptive and Chi-squared statistics were applied to observe most prevalent type of Vertucci's classification and to study its association between genders.

Results: Type I root canal configuration was identified as most common type in mandibular first premolars of right side [males: 36(87.8\%); females: 65(100\%)] and left side [males: 37(90.2\%); females: 65(100\%)]. In mandibular second premolar Type I was commonly observed in right as well as left side [males: $34(82.9 \%)$, females: 60(92.3\%)]. Type $\vee 3$ (3.8\%) was the second most common type followed by Type IV 1 (100\%). The significant difference was observed in both males and females mandibular first premolars right and left sides $(p=0.016, p=0.01$ respectively).

Conclusion: Type-I root canal configuration in mandibular first premolars of right side was found the most common in the targeted population. We also found that mandibular first and second premolars are single-rooted and have single ovoid canal.

Keywords: Mandibular Premolars; Cone Beam Computed Tomography; Gender; Sexual Differentiation; Root Canal Configuration.

\section{Corresponding Author:}

Dr. Muhammad Bilal Bashir

Department of Oral Biology,

Dr. Ishrat-Ul-Ebad Khan Institute of Oral Health Sciences (DIKIOHS),

Dow University of Health Sciences,

Karachi, Pakistan.

Email: drbilalmds@gmail.com

https://doi.org/10.36283/PJMD10-4/007

\section{INTRODUCTION}

The morphology of a root canal plays a vital role in the endodontic treatment of teeth. The roots and their configuration differ in different teeth'. Therefore, a comprehensive knowledge of their anatomic dissimilarities may help clinicians to perceive those deviations during root canal therapy, enhancing the chance for successful treatment ${ }^{2}$. Overlooking to locate, prepare and obturate all the canals may cause failure in treatment. The root and canal anatomy may vary in a different ethnic or regional population ${ }^{3-4}$. It is essential to be aware of deviations in tooth morphology and distinguishing features in 
different ethnic groups as such, information can assist in locating, negotiating and managing the canals ${ }^{5}$. It has been stated that the original geometry of a canal, before shaping and cleaning procedures, had more influence on the changes, which occurred during preparation, than the instrumentation technique itself 6 .

Conventional radiographic techniques such as periapical radiographs are used commonly in clinical practice to identify the configuration of canals ${ }^{7}$. However, the 2D imaging of the canals sometimes results in missed canals and other anatomical structures, which may lead to reinfection and failure of root canal treatment ${ }^{8}$. The competent calculation of the anatomic morphology before performing endodontic treatment should use the most precise imaging system. Various systems have been employed to study the internal anatomy of teeth in three dimensions including clearing and staining canal technique, cone beam computed radiography (CBCT), digital and contrast medium-enhanced radiographic techniques?, scanning electron microscope (SEM) and micro-computed radiography ${ }^{10}$.

CBCT in particular is a non-invasive and a sensitive method which provides the three-dimensional image of entire tooth and its supporting structure ${ }^{11}$. It is very valuable in dento-maxillofacial imaging. The non-invasive, CBCT scanning was introduced in the 1990s and has many dental applications, including morphologic analysis ${ }^{12}$. The variations in root canal configuration are influenced by genetic factors. Asian populations present one of the widest variations in coronal shape, external root form and internal canal space morphology ${ }^{13}$. The root canal system is intricate and the canal may branch, divide and re-join taking various pathways to the apex. Vertucci found numerous complex canal systems and identified eight pulp canal configurations ${ }^{14}$. Many other classification systems and modifications have been introduced but to-date Vertucci's system remains the most reliable method to identify the anatomical variations in the root canal system of different teeth. None of these studies has data related to the morphology of mandibular premolars of Pakistani population. Therefore, mandibular premolars have been selected for this study.

The aim of this study is to determine the most common type of root canal configuration of mandibular premolars and their frequency amongst males and females in sample size of Pakistani population. The data for the morphological variations in the root canal of mandibular premolars is limited. Therefore, mandibular premolars have been selected for this study. The study aimed to evaluate the Vertucci's classification to describe the canal configuration and root canal morphology of mandibular premolars in sample size of Pakistani population using CBCT.

\section{METHODS}

This is a cross sectional observational study, to evaluate the commonly found type of vertucci's classification and to compare the root canal morphology of mandibular premolars in males and females. The estimated population size was 432 mandibular premolar teeth of right and left side. Patients from Dow University of Health Sciences, both male and female with age ranging between 18-30 years were selected. Patients that reported to the dental OPD of Dow International Dental College, Dow University of Health Sciences were identified and selected according to the inclusion criteria. These patients were referred to radiology department for the need of $\mathrm{X}$-rays having either third molar impaction or caries in different teeth but not involving mandibular premolars. Patients having caries or restored mandibular premolars, having periodontal abscess, fractured mandibular premolars or any other root anomaly were excluded. Rotograph Evo 3D and 3D planner version 2.0_2018 $8^{\circ}$ villa SM 2018 software was used. The convenience sampling technique was used to identify and recruit the patients for the study. The participants were explained the steps involved in the procedure. After taking informed consent from the subjects, their socio-demographic details and medical and dental history were obtained.

The patients were then referred to the Radiology department where the CВCT images were obtained using the $\mathrm{CBCT}$ equipment. The subjects were provided neck collars and lead aprons and as low as reasonably achievable (ALARA) principle was followed. After obtaining the CBCT images, the number of the roots, position of the root, canal bifurcation and Vertucci's classification of root canals was examined with the help of 3D planner version 2.0_2018 villa SM 2018 by two examiners at two different times. The inter-examiner reliability was calculated for the observed parameters and found out to be highly significant.

The data analysis was executed using SPSS v21. Chi-squared test was applied between classification and mandibular first and second premolar to identify the evaluation and compare the differences in the mandibular first and second premolar between males and females. The data was non-parametric and sample size was less than 1000, therefore, Fisher Exact test was used for comparison. Using PASS $\mathrm{v} 11$, test for 01 sample proportion with $95 \%$ confidence interval, $80 \%$ power of the test, $5 \%$ margin of error and prevalence of $62.84 \%$ of type I classification.

\section{RESULTS}

The descriptive statistics were applied to calculate the 
mean age of the participants and gender. The mean age of the study participants was $23.3 \pm 4.3$ years. There were 43 males and 65 female patients. The total number of teeth observed on the right and the left side are 432 of which $48.8 \%$ ( $n=211$ ) first premolars and $49 \%(n=212)$ second premolars had one canal and $1.1 \%(n=5)$ first premolars and $0.9 \%(n=4)$ second premolars were found to have two canals. The two-canal morphology of the premolars was only observed in males therefore no statistics could be computed for them. No variation was observed in the number of roots. All the premolars that were observed were single rooted.
The Chi-square statistics were applied to check the association of the Vertucci's classification in mandibular first and second premolars bilaterally. Type I was the most commonly observed Vertucci's classification in mandibular premolars of both sides followed by Type $\mathrm{V}$. Type IV was the least commonly observed classification (Table 1). No other Vertucci's type was observed in the premolars. Statistically significant association was observed in the mandibular first and second premolar on the right $(p<0.001)$ and left side $(p<0.001)$. The values obtained by Fisher's exact test were used, as the cell count was less than 20. Table 1 shows the association when one particular type was compared to another type.

Table 1: Association of Vertucci's Classification in mandibular first and second premolar's right side and left side.

\begin{tabular}{|c|c|c|c|c|c|}
\hline \multicolumn{6}{|c|}{ Mandibular First and Second Premolar's Right Side } \\
\hline \multirow{2}{*}{ First Premolar } & \multicolumn{3}{|c|}{ Second Premolar } & \multirow[b]{2}{*}{ Total } & \multirow[b]{2}{*}{$p$-Value } \\
\hline & $\begin{array}{l}\text { Type I } \\
\text { n (\%) }\end{array}$ & $\begin{array}{c}\text { Type IV } \\
\text { n (\%) }\end{array}$ & $\begin{array}{c}\text { Type V } \\
\text { n (\%) }\end{array}$ & & \\
\hline Type I & $92(91.1 \%)$ & $4(4 \%)$ & $5(5 \%)$ & 101 & \multirow{3}{*}{$<0.001^{* *}$} \\
\hline Type IV & $1(100 \%)$ & $0(0 \%)$ & $0(0 \%)$ & 1 & \\
\hline Type V & $1(88.7 \%)$ & $0(0 \%)$ & $3(3.8 \%)$ & 4 & \\
\hline \multicolumn{6}{|c|}{ Mandibular First and Second Premolar's Left Side } \\
\hline Type I & $93(98.9 \%)$ & $0(0 \%)$ & $1(1.1 \%)$ & 94 & \multirow{3}{*}{$<0.001^{* *}$} \\
\hline Type IV & $4(100 \%)$ & $0(0 \%)$ & $0(0 \%)$ & 4 & \\
\hline Type V & $5(62.5 \%)$ & $0(0 \%)$ & $3(37.5 \%)$ & 8 & \\
\hline
\end{tabular}

**p-value less than 1\% as computed by Fisher's exact test.

Table 2: Frequency of Vertucci's classification in mandibular premolars (both sides) between males and females.

\begin{tabular}{|c|c|c|c|c|c|}
\hline \multirow[b]{2}{*}{ Mandibular Premolar Sides } & \multicolumn{3}{|c|}{ Vertucci's Classification } & \multirow[b]{2}{*}{ Total } & \multirow[b]{2}{*}{ p-Value } \\
\hline & $\begin{array}{l}\text { Type I } \\
\text { N (\%) }\end{array}$ & $\begin{array}{c}\text { Type IV N } \\
\text { (\%) }\end{array}$ & $\begin{array}{c}\text { Type V } \\
\text { N (\%) }\end{array}$ & & \\
\hline \multicolumn{6}{|c|}{ Mandibular First Premolar (Right Side) } \\
\hline Males & $36(87.8 \%)$ & $1(2.4 \%)$ & $4(9.8 \%)$ & 41 & \multirow{2}{*}{$0.016^{*}$} \\
\hline Females & $65(100 \%)$ & $0(0 \%)$ & $0(0 \%)$ & 65 & \\
\hline \multicolumn{6}{|c|}{ Mandibular Second Premolar (Right Side) } \\
\hline Males & $34(82.9 \%)$ & $3(7.3 \%)$ & $4(9.8 \%)$ & 41 & \multirow{2}{*}{0.23} \\
\hline Females & 60 (92.3\%) & $4(3.8 \%)$ & $8(7.5 \%)$ & 65 & \\
\hline \multicolumn{6}{|c|}{ Mandibular First Premolar (Left Side) } \\
\hline Males & $37(90.2 \%)$ & $0(0 \%)$ & $4(9.8 \%)$ & 41 & \multirow{2}{*}{$0.01^{*}$} \\
\hline Females & 65 (100\%) & $0(0 \%)$ & $0(0 \%)$ & 65 & \\
\hline \multicolumn{6}{|c|}{ Mandibular Second Premolar (Left Side) } \\
\hline Males & 34 (82.9\%) & $3(7.3 \%)$ & $4(9.8 \%)$ & 41 & \multirow{2}{*}{0.23} \\
\hline Females & 60 (92.3\%) & 1 (1.5\%) & 4 (6.2\%) & 65 & \\
\hline
\end{tabular}

*p-value significant at $5 \%$ as computed by Fisher's exact test. 
In males, Type I was commonly observed in mandibular premolars. In the right mandibular first premolar $87.8 \%$ teeth had the Type I configuration $(n=36)$, followed by Type $V$ which is $9.8 \%(n=4)$ and $2.4 \%$ of Type $\mathrm{VI}$ configuration $(\mathrm{n}=1)$ in males. Type was the only type that was observed in mandibular first premolars of right side in females. Statistically significant differences were observed between right mandibular first premolars of males and females $(p-=0.016)$ (Table 2). The Type । classification was a common observation in right mandibular second premolars in both males and females $82.9 \%(n=34)$ and $92.3 \%(n=60)$ respectively. Type IV was observed in $7.3 \%$ males $(n=3)$ and $3.8 \%$ of females $(n=4)$. Type $V$ was observed in $9.8 \%$ males $(n=4)$ and $7.5 \%$ of females $(n=8)$. No significant difference was observed between males and females ( $p$-value $=0.23$ ).

In the left mandibular first premolars, Type I was the only type observed in females. In males $90.2 \%$ teeth had Type I ( $n=37)$ followed by Type $\vee$ which was $9.8 \%(n=4)$. Type IV was not observed in males. Statistically significant association was found between males and females ( $p$-value $=0.01$ ). In the left mandibular second premolars Type I was observed in $82.9 \%(n=34)$ of males and $92.3 \%(n=60)$ of females. Type IV was observed in $7.3 \%(n=3)$ of males and $1.5 \%(n=1)$ of females. While type $\vee$ was observed in $9.8 \%$ of males $(n=4)$ and $6.8 \%$ of females $(n=4)$. No significant was observed between males and females.

The coronal view (Figure 1) of the teeth that were used to observe the shape of orifice, which was ovoid in all mandibular premolars and no exceptions, were observed. The sagittal view of the tooth and these images were used to identify the Vertucci's classification in the mandibular premolars (Figures $2 a$ and b).

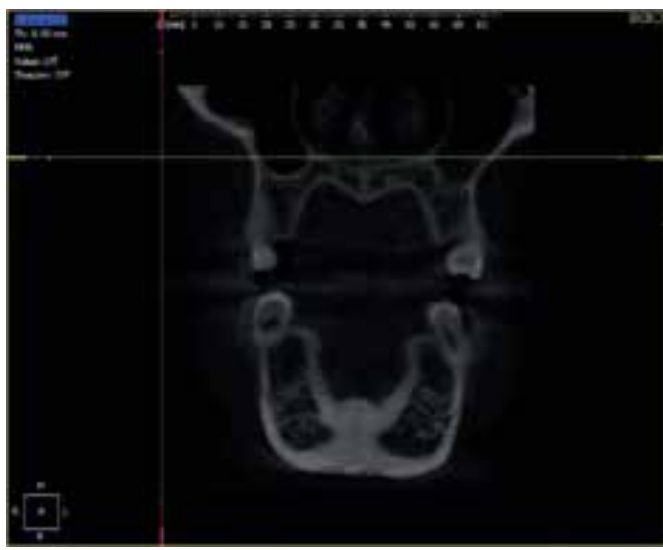

Figure 1: The coronal view of the cone beam computed tomography (CBCT) image showing the shape of the orifice of the canals of the teeth.

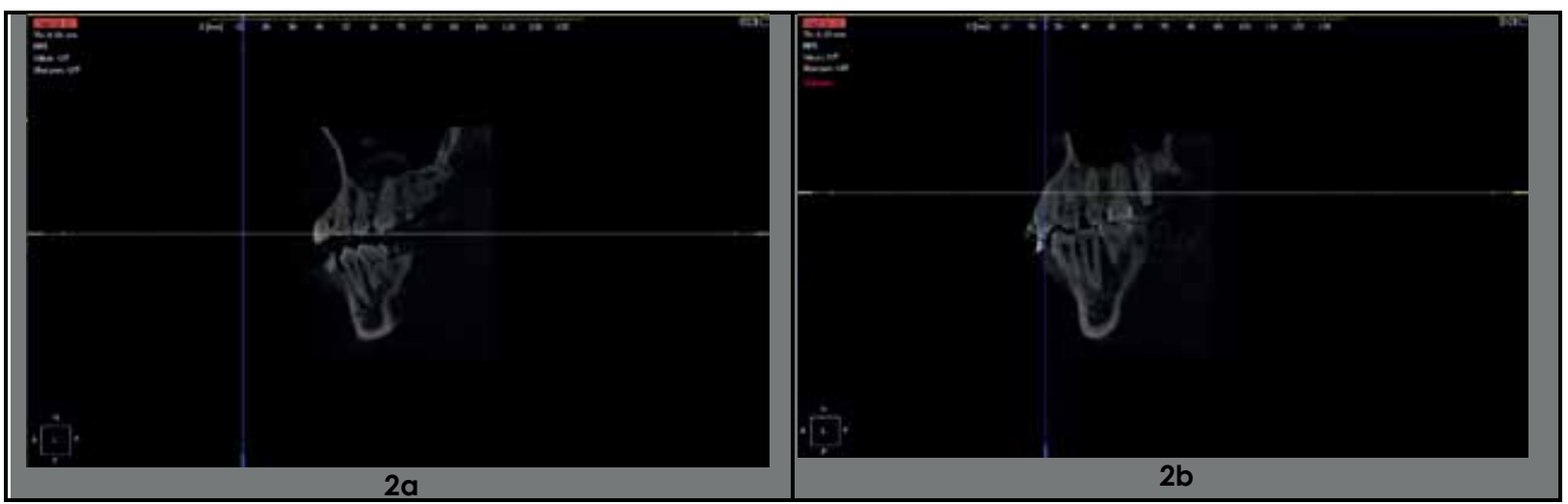

Figure 2a: The left mandibular premolar showing Type I canal configuration in the sagittal view; 2b: The sagittal view of the mandibular premolar showing the Type $V$ configuration of the canal.

\section{DISCUSSION}

The most common type of root canal configuration in our study was Type I that is a single canal from the start of the pulp chamber to the apical foramen.
Next common type that was observed was type $\mathrm{V}$, a single canal starting from the pulp chamber and dividing into two separate canals at the apical part of tooth. Type IV, that is presence of two canals with two separate openings was also observed in our 
study. Other canal configurations were not observed. Many patients involved in the study had one root and type I configuration in mandibular first (96.4\%) and mandibular second premolar (95.6\%) ${ }^{15}$. As per study conducted in Saudi Arabia $79.2 \%$ of females had single canal configuration, whereas males had $54.1 \%$ single canal, respectively ${ }^{16}$.

The study conducted on Western Chinese population observed single rooted first premolars (98\%) and $87.1 \%$ had single canal. They observed type I as most common configuration followed by type $V^{12}$. The presence of single canal have been observed to be $69.3-86 \%$ in many studies, two canals have been reported rarely ${ }^{4}$. In mandibular first premolar type I was (62.2\%) followed by type $V$ (20.3\%), type III (10.9\%), type VI (4.2\%) and type II, IV and VII respectively, whereas mandibular second premolar had (78\%) type I ${ }^{17}$. While in our sample, it was not observed. Studies conducted on Indian population also observed higher prevalence of Type I classification and single rooted teeth ${ }^{18}$. The study conducted on Saudi population observed single rooted teeth with single canal and prevalent type I ${ }^{19}$. In study conducted on Turkish Cypriots, 954 mandibular premolars were observed and $92.8 \%$ teeth were noted to have single root with one cana $^{20}$. Type I followed by type $V$ was the most common morphology observed in their study and type IV and II were least common. Another study which compared the clearing technique and CBCT in mandibular premolars observed Type I followed by Type $V$ and IV ${ }^{21}$. In our study, the percentage of mandibular premolars with two canals was very low $1.1 \%$ and $0.9 \%$, respectively) and was only observed in males. The findings in our study are in accordance with other studies using similar technique.

The significant difference in males and females was observed in mandibular first premolars on both sides. The results of this study show that gender dimorphism occurs in the canal configurations. Mandibular first premolar found to have (98.8\%) single canal with (81\%) type I configuration in Chinese population ${ }^{22}$. It was observed in our study that females had a higher percentage of Type । prevalence in both types of premolars on each side as compared to males. As per few studies it showed type I configuration to be more prevalent $155.3 \%$ $99.6 \%)$ having single root $(89.5 \%-100 \%)^{23}$.

There are numerous methods for assessment of root canal anatomy ${ }^{24,25}$. Using CBCT pictures has many benefits, such as having outcomes similar to those of direct procedures ${ }^{25}$. While taking single CBCT image a greater area is added sometimes resulting in recording adjacent dentition as well ${ }^{26}$. This may benefit in a precise identification of type of the tooth in few cases. In comparison to CBCT images and regular 2-Dimensional X-ray images, it is found to have given better accurate results that led to more successful outcome of the treatment ${ }^{27,28}$.

Mandibular premolars have always been a challenge for endodontists due to their diverse root canal anatomy, and a lot of work has already been done on it ${ }^{29}$. Still a lot more work is pending and need to be done for the better interpretation and hence better treatment by practitioners. Study shows that rate of formation and completion of tooth roots is influenced greatly by genetic and hormonal factors and can contribute greatly to gender dimorphism between the roots of the teeth ${ }^{30}$.

The study is first of its kind to be conducted on mandibular premolars of Pakistani population using CBCT. This will provide baseline data for further researches to be conducted on morphological variations. One of the limitations of CBCT is that it can only determine limited contrast resolution, mainly due to comparatively high-distributed emission during image attainment and innate flat panel detector associated artefacts. The technology is relatively expensive; patients have limited knowledge of its benefits. Further studies should be conducted on larger sample size and ethnic variations should be identified not only on mandibular premolars but on other teeth as well. Combination of different techniques such as clearing technique, $2 \mathrm{D}$ radiographs and $\mathrm{CBCT}$ should be compared to evaluate the morphological variations of root canal anatomy.

\section{CONCLUSION}

It has been observed in this study that mandibular first and second premolars are single-rooted and have single ovoid canal. The incidence of a single canal was high in mandibular premolars as compared to two canals. No mandibular premolars with two canals were reported in females. Type I root canal configuration was identified as the most common type in mandibular $1^{\text {st }}$ premolars of right side. Type $\vee$ was the second most common type followed by Type IV. These findings are in accordance to the studies conducted by other researchers using $\mathrm{CBCT}$ in other population-based studies.

\section{ACKNOWLEDGMENTS}

The authors would like to acknowledge the Dow University for facilitating the study.

\section{CONFLICT OF INTEREST}

The authors declare no conflict of interest. 


\section{ETHICS APPROVAL}

The ethical approval was obtained from the Institutional Review Board (IRB) of Dow University of Health Sciences having reference number: IRB-1301/DUHS/Approval/2019/53.

\section{PATIENT CONSENT}

The participants were explained the steps involved in the procedure and consents were obtained.

\section{AUTHORS' CONTRIBUTION}

MBB was the primary author, collected the data, did the interpretation and analysis, and wrote the manuscript. IA helped in selecting the topic, re-analysed the data for inter-examiner reliability. Both the authors contributed in write-up and final revision of the manuscript.

\section{REFERENCES}

1. Alrahabi M, Zafar MS. Evaluation of root canal morphology of maxillary molars using cone beam computed tomography. Pak J Med Sci. 2015; 31 (2):426-430.

2. Dinakar C, Shetty UA, Salian VV, Shetty P. Root canal morphology of maxillary first premolars using the clearing technique in a south Indian population: An in vitro study. Int J Appl Basic Med Res. 2018;8(3):143-147. 3. Amos ER. Incidence of bifurcated root canals in mandibular bicuspids. J Am Dent Assoc. 1955;50(1): 70-71.

4. Vertucci FJ. Root canal morphology of mandibular premolars. J Am Dent Assoc. 1978;97(1):47-50.

5. Velmurugan N, Sandhya R. Root canal morphology of mandibular first premolars in an Indian population: A laboratory study. Int Endod J. 2009;42(1):54-58.

6. FJ V. Root canal morphology and its relationship to endodontic procedures. Endod Topics. 2005; 10(1):3-29.

7. Al-Nazhan S, Al-Daafas A, Al-Maflehi N. Radiographic investigation of in vivo endodontically treated maxillary premolars in a Saudi Arabian sub-population. Saudi Endod J. 2012;2(1):1-5.

8. Zhang D, Chen J, Lan G, Li M, An J, Wen X, et al. The root canal morphology in mandibular first premolars: a comparative evaluation of cone-beam computed tomography and micro-computed tomography. Clin Oral Investig. 2017;21 (4):1007-1012.

9. Neelakantan P, Subbarao C, Subbarao CV. Comparative evaluation of modified canal staining and clearing technique, cone-beam computed tomography, peripheral quantitative computed tomography, spiral computed tomography, and plain and contrast medium-enhanced digital radiography in studying root canal morphology. J Endod. 2010;36(9):1547-1551.

10. Verma P, Love R. A Micro CT study of the mesiobuccal root canal morphology of the maxillary first molar tooth. Int Endod J. 201 1;44(3):210-217.

11. YU X, GUo B, Li KZ, Zhang R, Tian YY, Wang H, et al. Cone-beam computed tomography study of root and canal morphology of mandibular premolars in a western Chinese population. BMC Med Imaging. 2012;12(1):1-5.

12. Sherrard JF, Rossouw PE, Benson BW, Carrillo R, Buschang PH. Accuracy and reliability of tooth and root lengths measured on cone-beam computed tomographs. Am J Orthod Dentofacial Orthop. 2010;137(4):S100-S108.

13. Singh S, Pawar M. Root canal morphology of South Asian Indian maxillary molar teeth. Eur J Dent. 2015:9(01):133-144.

14. Vertucci FJ. Root canal anatomy of the human permanent teeth. Oral Surg Oral Med Oral Pathol Oral Radiol. 1984;58(5):589-599.

15. Alfawaz H, Alqedairi A, Al-Dahman YH, Al-Jebaly AS, Alnassar FA, Alsubait $S$, et al. Evaluation of root canal morphology of mandibular premolars in a Saudi population using cone beam computed tomography: A retrospective study. Saudi Dent J. 2019;31(1):137-142.

16. Algarni YA, Almufarrij MJ, Almoshafi IA, Alhayaza $\mathrm{HH}$, Alghamdi N, Baba SM. Morphological variations of mandibular first premolar on cone-beam computed tomography in a Saudi Arabian sub-population. Saudi Dent J. 2021;33(3):150-155.

17. Hajihassani N, Roohi N, Madadi K, Bakhshi $M$, Tofangchiha M. Evaluation of root canal morphology of mandibular first and second premolars using cone beam computed tomography in a defined group of dental patients in Iran. Scientifica. 2017;2017:1-7.

18. Singh S, Pawar M. Root canal morphology of South Asian Indian mandibular premolar teeth. J Endod. 2014:40(9):1338-1341.

19. Ozcan G, Sekerci AE, Cantekin K, Aydinbelge M, Dogan S. Evaluation of root canal morphology of human primary molars by using $\mathrm{CBCT}$ and comprehensive review of the literature. Acta Odontol Scand. 2016;74(4):250-258.

20. Celikten B, Orhan K, Aksoy U, Tufenkci P, Kalender $A$, Basmaci $F$, et al. Cone-beam CT evaluation of root canal morphology of maxillary and mandibular premolars in a Turkish Cypriot population. BDJ Open. 2016;2(1):1-5.

21. Khademi A, Mehdizadeh M, Sanei M, Sadeqnejad $\mathrm{H}$, Khazaei S. Comparative evaluation of root canal morphology of mandibular premolars using clearing and cone beam computed tomography. Dent Res J. 2017; 14(5): 321-325.

22. Di Wu DQ, Xin BC, Sun DG, Ge ZP, Su JY. Root canal morphology of maxillary and mandibular first premolars analyzed using cone-beam computed tomography in a Shandong Chinese population. Medicine. 2020; 99(20): 1-8.

23. Wolf TG, Anderegg AL, Wierichs RJ, Campus G. Root canal morphology of the mandibular second premolar: a systematic review and meta-analysis. BMC Oral Health. 2021;21(1):1-11. 
24. Sousa TO, Haiter-Neto F, Nascimento EH, Peroni LV, Freitas DQ, Hassan B. Diagnostic accuracy of periapical radiography and cone-beam computed tomography in identifying root canal configuration of human premolars. J Endod. 2017;43(7):1176-1179.

25. Nazeer MR, Khan FR, Ghafoor R. Evaluation of root morphology and canal configuration of maxillary premolars in a sample of Pakistani population by using cone beam computed tomography. J Coll Physicians Surg Pak. 2018;68(3):423-427.

26. Ordinola-Zapata $R$, Bramante $C M$, Versiani MA, Moldaver Bl, Topham G, Gutmann JL, et al. Comparative accuracy of the Clearing Technique, CBCT and Micro-CT methods in studying the mesial root canal configuration of mandibular first molars. Int Endod J. 2017:50(1):90-96.

27. Abarca J, Duran M, Parra D, Steinfort K, Zaror C, Monardes $\mathrm{H}$. Root morphology of mandibular molars: a cone-beam computed tomography study. Folia Morphol. 2020;79(2):327-332.

28. Maghfuri S, Keylani H, Chohan H, Dakkam S, Atiah A, Mashyakhy M. Evaluation of root canal morphology of maxillary first premolars by cone beam computed tomography in Saudi Arabian southern region subpopulation: An in vitro study. Int J Dent. 2019;2019:1-6.

29. Kongkiatkool P, Puapichartdumrong P, Tantanapornkul W, Piyapattamin T, Wisithphrom K. Accuracy of digital periapical radiography and cone beam computed tomography for evaluation of root canal configuration in human mandibular first premolars. J Int Dent Med Res. 2020;13(1):80-85.

30. Doumani M, Habib A, Alhalak AB, Al-Nahlawi TF, Al Hussain F, Alanazi SM. Root canal morphology of mandibular canines in the Syrian population: a CBCT assessment. J Family Med Prim Care. 2020; 9(2):552-555. 